\title{
Speech Production Quality of Cochlear Implant Users with Respect to Duration and Onset of Hearing Loss
}

\author{
Suzan Ruffa ${ }^{a}$ Tobias Bocklet ${ }^{b}$ Elmar Nöth ${ }^{b}$ Joachim Müller ${ }^{c}$ \\ Eva Hoster ${ }^{d}$ Maria Schuster ${ }^{c}$ \\ ${ }^{a}$ ORL Clinic Frankfurt/Oder, Frankfurt/Oder, ${ }^{b}$ Department of Computer Science, University \\ of Erlangen-Nuremberg, Erlangen, and ${ }^{\mathrm{C}}$ Department of Otorhinolaryngology, Head and \\ Neck Surgery and ${ }^{\mathrm{d}}$ Institute of Medical Informatics, Biometry, and Epidemiology, Ludwig- \\ Maximilians University, Munich, Germany
}

\section{Keywords}

Speech intelligibility $\cdot$ Automatic speech recognition · Adults $\cdot$ Cochlear implant

\begin{abstract}
Purpose: To assess whether postlingual onset and shorter duration of deafness before cochlear implant (CI) provision predict higher speech intelligibility results of $\mathrm{CI}$ users. Methods: For an objective judgement of speech intelligibility, we used an automatic speech recognition system computing the word recognition rate (WR) of 50 adult CI users and 50 age-matched control individuals. All subjects were recorded reading a standardized text. Subjects were divided into three groups: pre- or perilingual deafness $(A)$, both $>2$ years before implantation, postlingual deafness $<2$ years before implantation (B), or postlingual deafness $>2$ years before implantation (C). Results: CI users with short duration of postlingual deafness (B) had a significantly higher WR (median 74\%) than CI users with long duration of postlingual deafness $(C ; 68 \%, p<0.001)$ or pre-/perilingual onset $(A ; 56 \%, p<0.001)$. Compared to their control groups only $\mathrm{CI}$ users with short duration of postlingual deafness reached similar WR, others showed significantly lower WR. Other factors such as hearing loss onset, duration of CI use, or duration of amplified hearing showed no consistent influence on speech quality. Conclusions: The speech production quality of adult CI users shows dependencies on the onset and duration of deafness. These features need to be considered while planning rehabilitation.
\end{abstract}


Ruff et al.: Speech Production Quality of Cochlear Implant Users with Respect to Duration and Onset of Hearing Loss

\section{Introduction}

Hearing loss not only leads to restricted speech understanding, but might also alter speech production. Leder and Spitzer [1] revealed that even young people who developed normal speech before the onset of deafness showed speech distortions such as phonetic, voice, resonance, and prosodic alterations. As a consequence, the oral communication of people with hearing loss might be restricted because of diminished auditory abilities and additionally because of altered speech production.

Cochlear implants (CIs) are indicated in a profound to total loss when hearing aids do not provide sufficient speech perception. The CI replaces the mechanic-electric inner ear function and passes encoded electric impulses directly to the hearing nerve. Although speech perception results vary between CI users, in general, the implant enables good speech understanding, although the number of speech cues available to CI users is smaller than the number of speech cues available to people with normal hearing [2]. This might be due to the degraded signal provided by the CI or by CI users' inability to discriminate or process all the information that is actually transmitted by the implant. Some advances in speech perception could be reached by newer coding strategies [3, 4]. However, considering the different quality of electric representation of the acoustic environment with limited frequency representation provided by a $\mathrm{CI}$, auditory effects on the phonetic and spectral characteristics of speech can be expected in CI users. Yet, results are sometimes striking: even though present-day CIs are not designed to specifically deliver F0 information, which is a relatively strong acoustic cue for the voicing feature in people with normal hearing [5], CI users are capable of discriminating also small phonetic differences. Moreover, Teoh et al. [6] reported that $\mathrm{CI}$ users are able to distinguish between voiced and voiceless consonants.

In the following, we summarize the literature on speech production after cochlear implantation regarding different aspects of speech production and intelligibility. Furthermore, we refer to Guenther's theory on speech quality.

\section{Issues of Speech Production after Cochlear Implantation}

That CI provision changes users' speech production has long been known: the influence of better auditory feedback on the control of speech production concerning spoken vowels was detected in 1992 by Perkell et al. [7] when implants and speech encoding algorithms were less elaborate than nowadays. They examined $4 \mathrm{CI}$ users with profound deafness and found changes of vowel structures after implantation, but also variability between the subjects examined. TyeMurray and Kirk [8] investigated the vowel and diphthong production in a long-term study on 8 children: vowel and diphthong production became more accurate after implantation and in the follow-up intervals. Then, in 1994, Matthies et al. [9] examined 5 adults with profound hearing loss and demonstrated that especially those subjects with an incorrect or low contrast between the production of sibilants benefitted from their implant's ability to increase the distinctiveness of articulation. Furthermore, Lane et al. [10] described time- and frequencydependent features of speech: in a long-term study, 4 out of 5 CI users had increased voice onset time after implantation due to better auditory feedback; acoustic correlates of speech changed concurrently. In contrast, in a study on 7 adults with profound deafness, Lane et al. [11] found that hearing status after implantation had little effect on vowels referring to coarticulation. The vowels in the center of syllables had little formant changes after cochlear implantation. Perkell et al. [12] drew comparisons between the changes in different languages: in 14 adult subjects, speech changes after implantation showed differences between English and Spanish speakers, with greater variability in Spanish speakers, which is in accordance with Lane et al.'s results. Then, in 2003, Schenk et al. [13] reported that auditory feedback after cochlear implantation had a positive effect on the vowel production of 10 adult German-speaking CI users. 
CI users' voice, nasality, and prosodic features also change after implantation [14]. In contrast to a number of detailed descriptions of phonetic changes, the speech intelligibility of adult CI users is a rare topic of scientific reports. In one of the few works on speech intelligibility, Gould et al. [15] examined 11 adult CI users with postlingual deafness and found on average an increase in speech intelligibility, but also great variability of intelligibility among the subjects. This comes along with the variable results on other aspects of speech such as altered nasality, voice, formants, and prosody as described by some authors concerning speech outcomes after cochlear implantation [12, 16, 17]. Horga and Liker [18] compared several variables such as vowel formant space, voice versus voiceless differences, closure duration and voice onset time, word accent production, sentence stress production, voice quality, and pronunciation quality in three groups of 10 subjects each: children with a CI, children with profound deafness who used traditional hearing aids, and normal-hearing subjects. The results revealed that most CI users performed worse than people with normal hearing, but better than deaf subjects with hearing aids. One study of different possible factors influencing on speech revealed variable alterations in formant frequencies of vowels. The authors supposed that the reason for this variability might lie in the history of hearing, especially whether deafness occurred pre- or postlingually and how long it had lasted before CI provision [17]. Consequently, we expect that subjects' duration of deafness before implantation and age at the onset of deafness will impact their speech production outcomes.

\section{Intelligibility after Cochlear Implantation}

All the data mentioned so far were drawn from studies on small cohorts of CI users on details of speech and show inconsistent results. One study examined the speech of 181 subjects, but these subjects were older children and adolescents, not adults [19]. To the knowledge of the authors, until now general speech production abilities such as intelligibility of a bigger cohort of adult CI users have not yet been well described, although speech production cannot just be predicted with respect to the hearing status of the CI user or the implant's capacities of temporal and spectral resolution. Studies on larger cohorts are needed to identify aspects that might predict adult CI users' speech production outcomes.

\section{Theory of Articulation Quality: DIVA}

One crucial aspect of speech intelligibility is articulation. Precise articulation relies on precise auditory feedback. According to the DIVA theory (directions into velocities of articulators) [20], all speech sounds are represented in a "speech sound map" in the left operculum frontale. During speaking, this "speech sound map" is constantly compared to an "auditory state map" and "somatosensory state map." Deviations from the "speech sound map" are registered constantly in auditory or somatosensory "error maps" in order to adjust articulatory precision and velocity. In case of pre- or perilingual deafness, the "speech sound map" is restricted due to insufficient auditory perception. Correct adjustment of the articulation is therefore not possible. Also, postlingual deafness might lead to changes in these "maps," especially concerning the "auditory state map." Without functioning auditory feedback, there is no comparison between the "speech sound map," the "auditory state map," and "the error maps," thus leading to altered articulation [21]. In the DIVA model, removing speakers' auditory feedback will only slightly degrade speech in the short term because the feedforward subsystem and the sensorimotor state map that coordinate articulatory movements are still well tuned. However, if deafness continues for a long period, the feedforward command will slowly degrade. Considering articulation in normal-hearing individuals to represent a tradeoff between auditory clarity and minimal effort $[12,22]$, in deafened persons, the articulatory effort might be decreased when auditory feedback lacks. Moreover, if deafness occurs early in life, before the vocal tract has reached its adult shape, articulation might additionally be 
Ruff et al.: Speech Production Quality of Cochlear Implant Users with Respect to Duration and Onset of Hearing Loss

affected [12] as the sensorimotor state map does not fit anymore to adult vocal tract dimensions.

After cochlear implantation, the "auditory state map" is re-available for articulation adjustment but might not compensate completely due to still altered auditory feedback by a $\mathrm{CI}$ in comparison to normal hearing. Moreover, changes of the "speech sound map" during hearing loss and/or restricted articulatory effort after long lasting hearing loss might still be present. Moreover, according to Lazard et al., a long lasting auditory deprivation is a major factor predicting hearing performance after cochlear implantation of postlingually deafened CI recipients [23]. This might additionally prevent CI users with long lasting deafness to have sufficient auditory feedback.

In this study, we examined the relationship between speech production, assessed by automatic speech recognition (ASR) technique, and onset of deafness (pre-/peri- or postlingual) and duration of deafness before implantation in a cohort of $50 \mathrm{CI}$ users. Influences were examined on speech production intelligibility as the main function of speech: to make oneself understood. Until now, data on speech intelligibility of CI users have been rare. This might be due to the time- and manpower-intensive evaluation procedures used previously. Different methods can be applied to evaluate intelligibility [24]. Usually, intelligibility is judged perceptually, e.g., on Likert scales, or calculated as the percentage of correctly understood (or transliterated) words of a speech corpus [25]. Recently, a new method for the evaluation of speech quality was established that uses ASR [26]. In our application, it is mainly based on phonetic features, thus referring to the DIVA theory. The quotient of automatically evaluated correctly pronounced words (word recognition rate [WR]) corresponds to the perceptually evaluated speech intelligibility and is therefore called "intelligibility degree" as it is still not similar to intelligibility. It enables researchers to examine speech quality with a standardized speech corpus with perfect stability of ratings [27, 28].

In this study, the influence of the onset of deafness and of the duration of deafness before implantation as well as other factors regarding speech production quality was evaluated.

\section{Subjects and Methods}

\section{Subjects}

In this study, CI users up from the age of 16 were included during regular out-clinic treatment. All were provided with one or two CIs from different brands.

The study was approved by the local ethics committee. After giving their informed consent, the study participants answered a standardized questionnaire regarding their history of hearing loss and performed a standardized speech recording. We asked for the onset of hearing loss (data might not be exact before the era of newborn hearing screening), the onset of deafness (defined as the time when hearing amplification was not sufficient for speech understanding in quiet anymore), and the duration of hearing amplification. The time of CI use, the number of CIs, and the age at implantation use were known from the documented patient histories.

Adults without a history of hearing loss or speech disorder were asked to participate as control individuals. They were patients who were treated in the clinic for other reasons than hearing or speech issues, or relatives of patients.

For the CI users, the implantation procedure including surgery, fitting, and rehabilitation followed a standardized protocol: 4-6 weeks after cochlear implantation, the speech processor is activated; fitting is performed on two following days. Four weeks later, the next fitting session takes place together with speech and language therapy (1-day procedure). Regular appointments are scheduled 3 months, 6 months, and 1 year after surgery. These sessions are accompanied by hearing training. Afterwards, regular controls are performed at least once a year.

CI users were divided into three groups, according to their onset of deafness and the duration of functional deafness before CI provision: (1) Group A: pre- and perilingual deafness; received CI >2 years after hearing aids no longer provided functional speech understanding. (2) Group B: postlingual deafness; 
Ruff et al.: Speech Production Quality of Cochlear Implant Users with Respect to Duration and Onset of Hearing Loss

received CI within 2 years after hearing aids no longer provided functional speech understanding. (3) Group C: postlingual deafness; received $\mathrm{CI}>2$ years after hearing aids no longer provided functional speech understanding.

Speech Corpus

For speech production evaluation, each subject once read a standardized speech test of 97 German single words including all German phonemes in different positions in the word. As we mainly concentrated on phonetic features according to the DIVA theory, we decided for single words. However, some other aspects of intelligibility, such as prosody, were not taken into account.

The words were presented on a monitor. The subjects' speech was recorded with a headset-mounted microphone (Beyerdynamic Microphone Opus 54.15-3) using the SpeechRecorder program of the Institute for Phonetics and Speech Processing from the University of Munich with a sampling frequency of $22.05 \mathrm{kHz}$. Recordings were performed in a noise-absorbent room usually used for audiology testing. Recording quality was perceptually controlled throughout the recordings. The subjects were asked to repeat words when reading errors occurred. During the recordings, the instructor took care that the subject read in normal intensity and that recordings were not over- or underamplified based on a graphical display of the speech energy. The sound pressure level of the spoken words was determined on the 10 central words using Praat.

\section{ASR System}

A well-tested ASR system developed at the Department of Pattern Recognition at the University of Erlangen-Nuremberg was used to evaluate speech. The software was applied via a client-server network on commonly used computers.

The speech recognizer is based on semicontinuous hidden Markov models with polyphones, a hierarchical representation of allophonic subword units. The polyphone concept generalizes the biphone or triphone idea with an (in theory) arbitrarily large phonetic context [29]. Mel-frequency cepstral coefficients (MFCCs) were used as acoustic frontend. MFCCs employ a windows size of $16 \mathrm{~ms}$ and are extracted every $10 \mathrm{~ms}$. The feature vector consists of the short-time energy and 11 MFCCs. Additionally, the parametric representation was enriched by 12 delta coefficients computed as the slope of the regression of five consecutive windows.

Neither the order of words nor syntactic information were included in the evaluation as only single words were read [30]. Normal-hearing adults from all over Germany served as the training population to cover most dialectal regions. All speakers were, however, asked to speak standard German. Ninety percent of the training population (304 males, 274 females) were younger than 40 years. From the VERBMOBIL German data, we used $27 \mathrm{~h}$ of speech (consisting of 11,714 utterances and 257,810 words) for training. The vocabulary of the recognition system was substituted by the 97 words of the applied speech test. For the evaluation of the degree of speech intelligibility, WR was computed as the percentage of correctly recognized words of the spoken 97 words.

In previous evaluations on speech, the applied method using WR as a measure of speech intelligibility degree resulted in a very high consistency to the perceptually evaluated intelligibility of a panel of listeners on a sentence-based evaluation, on single words and on transcriptions of spoken words (both $r$ and $\rho$ always $>0.8)[25,31-33]$.

\section{Statistics}

We compared the WR between the three groups as well as each group with its age-matched control group. The comparison of the WR between the three groups was performed using a Kruskal-Wallis test with a significance level of 5\%. If the Kruskal-Wallis test was significant, pairwise Mann-Whitney U tests were performed with the same significance level of 5\%, with no need of further adjustment for multiple testing due to a closed testing procedure. For each group, the comparison of the WR with its control group was done separately using a Mann-Whitney U test with a significance level of 5\%. No adjustment for multiple testing was performed here because these analyses were considered to answer different research questions.

We also analyzed the potential correlation of age, gender, age at deafness and duration of deafness, and duration of amplified hearing with WR. For group comparisons, Mann-Whitney U tests were computed; correlation analyses were performed using Spearman rank correlation. These correlation analyses were performed for the whole group of CI users group as well as selectively for CI users with postlingual hearing loss (groups B and C) and selectively for CI users with long duration of hearing loss (groups A and C). Nonparametric methods were used because WR was not normally distributed. Statistical analyses were performed using Excel 12.3.2. and SPSS Statistics 21. 
Table 1. Data of the cochlear implant user groups

\begin{tabular}{llll}
\hline & Group A & Group B & Group C \\
\hline Total number & 14 & 15 & 21 \\
\hline Women/men & $11 / 3$ & $11 / 4$ & $11 / 10$ \\
\hline Age, years & $37.4 \pm 17.9$ & $53.3 \pm 18.4$ & $59.1 \pm 14.7$ \\
& $33(18-71)$ & $60(17-78)$ & $55(38-82)$ \\
\hline Age at hearing loss onset, years & $1.8 \pm 2.5$ & $32.6 \pm 17.9$ & $25.6 \pm 18.3$ \\
& $0(0-4)$ & $38(7-60)$ & $25(4-65)$ \\
\hline Age at deafness onset, years & $18.7 \pm 21.7$ & $45.2 \pm 22.9$ & $46.1 \pm 22.7$ \\
\hline Duration of hearing amplification, years & $25(0-65)$ & $59(15-77)$ & $46(14-77)$ \\
\hline Duration of CI use, years & $29.4 \pm 17.7$ & $20.0 \pm 16.1$ & $21.6 \pm 11.5$ \\
& $29(1-67)$ & $21(0-29)$ & $19(5-41)$ \\
\hline Unilateral/bilateral implantation & $7.1 \pm 6.1$ & $6.0 \pm 5.2$ & $5.3 \pm 5.9$ \\
\hline Bimodal: CI and hearing aid & $5(0.5-19)$ & $4(1-17)$ & $4(0.5-18)$ \\
\hline
\end{tabular}

Values are presented as $n$ or as mean \pm standard deviation and median (range). Group A, B, and C were formed according to onset and duration of deafness, onset of hearing loss, and duration of hearing amplification by common hearing aids. Please note that the difference between age at hearing loss onset, duration of hearing amplification, and age of deafness do not match perfectly according to the group classification. This is due to the fact that some CI users still use a hearing aid on one ear. The history of hearing loss was assessed by a questionnaire and might lack precision, e.g., hearing loss onset was hard to determine before the introduction of newborn hearing screening. Onset of deafness was defined as the age when hearing amplification was not sufficient for speech understanding anymore. CI, cochlear implant.

\section{Results}

The study participants consisted of 100 adults. Fifty out of the 100 subjects were deaf and were CI users since at least 6 months. The group of CI users consisted of 33 women and 17 men aged $48.6 \pm 20.0$ years with uni- or bilateral CIs. All subjects were native speakers of German. The CI users had implants of different generations and from different manufacturers. The CI users' data including age, gender, history of hearing, duration of $\mathrm{CI}$ use, and duration of amplified hearing before implantation are given in Table 1 . The sound pressure level of the words spoken was measured on the central 10 words of each recording per subject, resulting in $64.4 \pm 3.67 \mathrm{~dB}$.

Fifty subjects with normal hearing and no apparent speech disorders were included as a control group. As age has an effect on articulatory precision, the controls were age-matched to the groups of CI users: control group A consisted of 14 subjects aged $39.6 \pm 9.0$ years, control group B of 15 subjects aged $55.9 \pm 17.8$ years, and control group C of 21 subjects aged $53.1 \pm 14.6$ years. They used their normal (and hence unaided) hearing when completing the test procedures.

\section{WR and Influencing Factors}

The correlation analysis concerning the influences on WR in the cohort of $50 \mathrm{CI}$ users is summarized in Table 2. The age as well as gender of the subjects showed no significant 
Ruff et al.: Speech Production Quality of Cochlear Implant Users with Respect to Duration and Onset of Hearing Loss

Table 2. Correlation analysis between word recognition rate and subject characteristics

\begin{tabular}{|c|c|c|c|c|c|c|c|c|}
\hline & Age* & Gender $^{+}$ & $\begin{array}{l}\text { Age at } \\
\text { deafness* }\end{array}$ & $\begin{array}{l}\text { Duration } \\
\text { of deafness* }\end{array}$ & $\begin{array}{l}\text { Duration } \\
\text { of CI use* }\end{array}$ & $\begin{array}{l}\text { Duration of } \\
\text { amplified } \\
\text { hearing* }\end{array}$ & $\begin{array}{l}\text { Number } \\
\text { of } \mathrm{CIs}^{+}\end{array}$ & $\begin{array}{l}\text { Bimodal } \\
\text { use }^{+}\end{array}$ \\
\hline \multirow[t]{2}{*}{ All $(n=50)$} & -0.04 & & 0.27 & -0.386 & 0.07 & -0.23 & & \\
\hline & 0.72 & 0.35 & 0.06 & 0.006 & 0.629 & 0.13 & 0.93 & 0.19 \\
\hline A and C (pre-/perilingual & 0.14 & & 0.21 & -0.24 & 0.02 & -0.29 & & \\
\hline $\begin{array}{l}\text { vs. postlingual long duration } \\
\text { of deafness, } n=35 \text { ) }\end{array}$ & 0.44 & 0.71 & 0.22 & 0.17 & 0.92 & 0.11 & 0.25 & 0.25 \\
\hline \multirow{2}{*}{$\begin{array}{l}\text { B and C (short vs. long } \\
\text { duration of postlingual } \\
\text { deafness, } n=36 \text { ) }\end{array}$} & -0.06 & & 0.067 & -0.395 & 0.12 & -0.02 & & \\
\hline & 0.72 & 0.28 & 0.7 & 0.017 & 0.47 & 0.91 & 0.86 & 0.14 \\
\hline
\end{tabular}

CI, cochlear implant. * Spearman $\rho, p$ value. ${ }^{+}$Mann-Whitney U test, $p$ value.

influence on WR in CI users. WR showed a significant relationship with the duration of deafness for all patients and for CI users with postlingual deafness.

\section{Group Comparisons}

The median WR for all CI users was 69\% (range 29-82\%). The Kruskal-Wallis test showed a significant difference in WR between groups A, B, and C regarding onset of deafness $(p=$ $0.026)$ and duration of deafness $(p=0.004)$. WR displayed high variation, particularly in groups $\mathrm{A}$ and $\mathrm{C}$, containing subjects with $\mathrm{CI}$ provision after $>2$ years of deafness. Median WR was highest in group B (74\%), whose subjects were provided with a CI within 2 years after postlingual deafness (Table 3). The WR of group A (56\%) and C (68\%) was significantly lower than that of group B (Mann-Whitney U test: A vs. B $p=0.002$, B vs. C $p=0.025$ ). Between groups A and C, WR showed no significant difference (Mann-Whitney U test: $p=0.25$ ). The WR of control group A was significantly different from that of control group B ( $p=0.004)$ and control group C ( $p=0.006)$ (Mann-Whitney U test) (Fig. 1).

Comparing each group with its age-matched control group, we found significant differences for groups A (56 vs. 82\%, Mann-Whitney U test, $p<0.001$ ) and C (68 vs. 77\%, MannWhitney U test, $p=0.001$ ). Group B subjects, who were provided with a CI within 2 years after deafening, showed scores rather similar to those of the control subjects (74 vs. $75 \%$, MannWhitney U test, $p=0.52$ ) (Table 3).

Other possibly influencing factors did not show a significant relationship with WR (age at hearing loss onset, duration of $\mathrm{CI}$ use, duration of amplified hearing).

\section{Discussion}

CIs are widely accepted as a most efficient technological resource for the restoration of hearing when hearing amplification is not sufficient for speech perception. In children with congenital deafness, Cosetti and Roland [34] showed in a review that early cochlear implantation can lead to normal speech acquisition. So, in general, CIs can enable deaf children to hear sufficiently in order to discriminate different phonemes and in order to develop normal speech production competence and intelligibility. However, previous evaluations concerning speech production with spectral analysis of phonemes showed variable results for adults 
Ruff et al.: Speech Production Quality of Cochlear Implant Users with Respect to Duration and Onset of Hearing Loss

Table 3. Word recognition rate of the cochlear implant users and the control group in the same age range of each group

\begin{tabular}{llll}
\hline & Group A & Group B & Group C \\
\hline CI group & $57.9 \pm 15.6$ & $73.6 \pm 5.2$ & $65.4 \pm 12.6$ \\
& $56(29-78)$ & $74(59-79)$ & $68(40-82)$ \\
\hline Control group & $82.3 \pm 6.1$ & $74.6 \pm 2.0$ & $75.1 \pm 7.8$ \\
& $82(72-82)$ & $75(62-83)$ & $77(62-90)$ \\
\hline CI versus control group & $p<0.001$ & $p=0.519$ & $p<0.001$ \\
\hline
\end{tabular}

Values are presented mean \pm standard deviation and median (range). Correlation analysis for CI versus control group was performed using the Mann-Whitney U test; the level of significance is given. CI, cochlear implant.

with postlingual deafness [15]. The literature on other features of speech also shows variable limitations of speech of adult CI users without identifying factors influencing on speech outcome.

Regarding the hearing outcome of adult CI users, some influencing factors could be identified in a large multicenter study, which indicated that a long duration of deafness is unfavorable for hearing after implantation [21]. The duration of deafness therefore might be also of importance for speech production outcome according to the DIVA theory [18]. In the present study on adult CI users' speech outcomes, we therefore focused on the role of duration of deafness and some other factors.

Fifty subjects provided with a CI were examined for the evaluation of speech quality. In order to investigate the influence of onset and duration of deafness on speech, we distinguished three groups of subjects. For group description in this study, we decided that 2 years were the critical duration of deafness for speech outcomes according to Neumeyer et al. [17]. In future studies including more postlingually deafened adults, we might more precisely identify a critical duration of deafness.

According to the history of hearing loss, we had different numbers of subjects in each group and different mean ages. Group A had the lowest mean age (37.7 years) as the group consisted of subjects who had already been hard of hearing during childhood. They were provided with a CI more than 2 years after hearing aids had not been sufficient for speech understanding anymore. Group C, the biggest group, was mostly comprised of CI users with a long history of deafness (mean onset at the age of 28 years). As the subjects in groups $\mathrm{A}$ and $\mathrm{C}$ were particularly used to impaired hearing, the decision for a CI might have been delayed. The high number of subjects in group $\mathrm{C}$ in comparison to group B might reflect the rather late implantation of deaf adults in Germany at the time of this investigation.

\section{Automatic Speech Recognition}

In this article, for speech evaluation, ASR adapted to adults' speech was applied for a medical purpose using a standardized reading text. ASR has been proven suitable for medical assessment for speech quality measurements [26-28, 31-33, 35, 36] and shows high agreement with perceptually judged intelligibility. It therefore stands for the ability to make yourself understood. When using no language model, ASR stands in for a standardized layman listener and can serve as a supporting means in medical speech assessment.

Before, for scientific purposes, speech evaluation procedures were usually based on the perceptual assessment of several experienced listeners, such as speech therapists, to form a 
Ruff et al.: Speech Production Quality of Cochlear Implant Users with Respect to Duration and Onset of Hearing Loss

Fig. 1. Data of 100 cochlear implant users with median and standard deviation of word recognition rate (WR) in the three patient groups and age-matched control groups in a box plot. The edges of the box are the 25 th and 75 th percentiles, the whiskers extend to the most extreme data points not considered outliers, and outliers are plotted individually and marked with circles. Group B with the highest WR values included subjects who lost hearing as adults and were provided with a cochlear implant within 2 years after hearing loss. Between groups $\mathrm{A}$ and $\mathrm{C}$ and their control groups, significant differences of WR were found (Mann-Whitney U test).

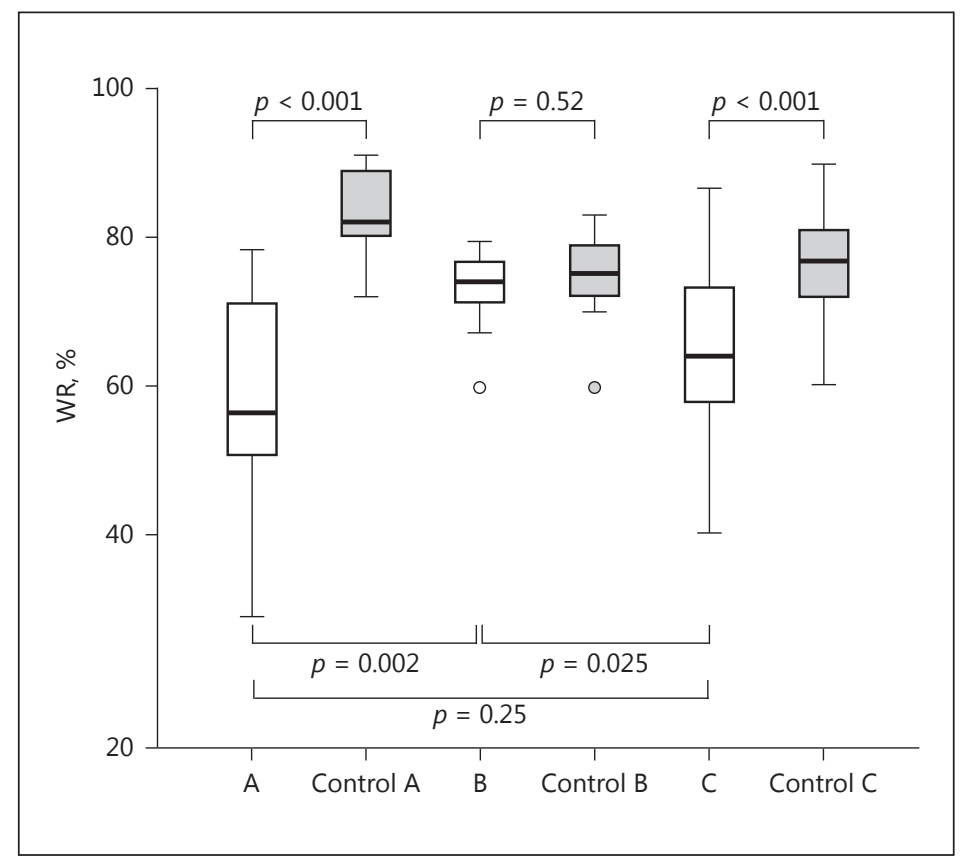

standard listener; results depended on the listeners' experience [31, 37]. Perceptual assessment procedures thus might have restricted reliability; moreover, they are very timeand manpower-consuming. We therefore decided to apply an objective tool that quickly allows for testing bigger cohorts with perfect comparability.

In accordance with most perceptual evaluations of intelligibility, the results of ASR assessment are mainly based on phonetic features. It shows low WRs for people with altered articulation skills and altered resonance, e.g., as shown before for children and adults with cleft lip and palate or oral cancer [27,31]. In previous investigations, ASR analysis and perceptually evaluated speech intelligibility showed agreement between 0.84 and 0.94 using Spearman rank correlation. The technique even allows for some more detailed analysis of altered speech: next to intelligibility assessment, even distinct alterations of phonetic features and of nasality in comparison to normal speech can be detected [38].

\section{Influences on WR}

In this investigation, we identified the duration and onset of deafness to be of significant importance for speech quality. Other factors such as age at hearing loss, duration of CI use, or duration of hearing amplification yielded no significant correlation with WR. Moreover, even the number of CIs or bimodal support (CI and hearing aid) showed no significant influence, although bilateral use of CIs has a positive influence on speech perception [36, 39, 40]. This was tested on the whole study group and also on the more homogeneous subgroups.

In previous articles, better speech perception outcome was associated with surgical variables such as deep insertion [41], cochleostomy [42], or techniques that aim to preserve lowfrequency hearing [43]. As the surgical procedures were similar in our study group, we would not expect them to play an eminent role in speech outcome.

\section{WR versus Onset and Duration of Deafness}

The ASR results measuring the WR showed a significant correlation with the onset of deafness (pre-/perilingual or postlingual) and the duration of deafness (less or more than 2 years). The results become clearer regarding the three groups (Table 3; Fig. 1). 
Ruff et al.: Speech Production Quality of Cochlear Implant Users with Respect to Duration and Onset of Hearing Loss

The WR scores in group B (postlingual deafness with short duration of deafness) were high and had a rather low variance. Early cochlear implantation after total to severe hearing loss preserved normal speech intelligibility in most subjects. In contrast, the WR scores of groups $\mathrm{A}$ and $\mathrm{C}$ were significantly lower than those of their control groups and showed wide variance. For group A, with pre-/perilingual deafness and late implantation, long-lasting effects on speech skills could be expected due to altered hearing during speech acquisition. When speech perception before or during speech acquisition is diminished, an altered "speech sound map" results, with incorrect adjustment to the "auditory state map." In accordance, An et al. [44] found that children with prelingual deafness without residual hearing had worse speech intelligibility than did children with postlingual deafness with residual hearing who were implanted before the sensitive period for language development ended. Because of missing auditory input at a critical phase, subjects in group A may not have been able to benefit from implantation in the same way as the subjects with postlingual deafness. Although CIs can stimulate developmental plasticity in the auditory brainstem even after many years of deafness in childhood, changes in the auditory cortex are limited. This might also be caused - at least in part - by the degree of reorganization which occurred during the period of deafness [45]. In accordance, a recent study using positron emission tomography showed differences in cerebral functions at several points after CI implantation between adults with pre- and postlingual deafness. Speech and speech-like stimuli showed different activation patterns on the left superior temporal gyrus, associated with speech comprehension, and on Broca's area [46]. Cochlear implantation in these patients may not totally compensate for earlier missing auditory effects on verbal speech skills and reorganization processes. Specialized speech training should be added to hearing training for CI users with low WR scores.

The mean WR scores of group $C$ were significantly lower and more variable than those of group B. Effects of deafness on speech, such as described by Leder and Spitzer [1], might persist even after cochlear implantation and increased auditory feedback on speech. As age might have a negative impact on WR scores [47] and as the CI groups were of different age ranges, we used age-adapted control groups. Concerning the control groups, the effect of age on WR is obvious, comparing control group A with the highest mean WR to the other two control groups which comprised older subjects. In contrast, among CI users we found no correlation between age and WR score. We suppose that age effects are insignificant due to influences of the onset of deafness on WR: although younger participants were found in group A, the effect of prelingual onset of deafness and long duration led to significantly lower WR. This effect is also clear regarding the mean WR scores of the control group.

Also, the duration of $\mathrm{CI}$ use after late implantation showed no significant influence in these CI users, although one might expect an influence as described by Peng et al. [48]. Tanamati et al. [49] showed that children with postlingual deafness can achieve high scores of intelligibility: after 10 years of CI use, 8 children received the highest scores and only 2 children received low scores. The effects of early hearing experiences might play a role and might also explain the variability of WR results in group A comprising CI users with pre- or perilingual deafness. Unfortunately, as the exact onset of deafness is hard to determine in patients who were born before newborn hearing screening was widely used, the effects of early hearing are hard to evaluate in adult CI users.

Our findings are in accordance with other comparisons of speech features of CI users with pre- and postlingual deafness: Neumeyer et al.'s [17] spectral examination of formant structures of CI users with different histories of hearing loss yielded higher alterations of the phoneme-determining formants F1 and F2 in subjects with prelingual deafness than in subjects with postlingual deafness. And according to Gould et al. [15], especially vowel intelligibility showed better outcomes in subjects with postlingual deafness (87-99\%) than in subjects with prelingual deafness (58-88\%). 
Ruff et al.: Speech Production Quality of Cochlear Implant Users with Respect to Duration and Onset of Hearing Loss

According to the results of the group comparisons, we found significant differences in the WR scores of groups A and C and their control groups. The group with the highest WR score, group B, showed no significant difference compared to the control group's result (73.6 vs. $74.2 \%$ ). Thus, group B was comparable to the normal population of the same age. This result underlines the need for early provision with one or two CIs after total hearing loss for pre-, peri-, and postlingual hearing loss in order to maintain or develop good speech production quality.

The data presented reveal how speech intelligibility is, in large part, shaped by an individual's hearing loss history. Furthermore, reliable data on adult CI users' speech production are important for rehabilitation strategies.

\section{Conclusions}

Increased auditory feedback after cochlear implantation is not always sufficient for good speech production quality in subjects with a long history of deafness and/or early onset of deafness. In contrast, CI provision soon after deafening increases the likelihood that a CI user can maintain good speech quality. Rehabilitation should especially focus on the speech production skills of CI users with deafness onset before or during speech acquisition and those who had a long period of insufficient or not sufficiently compensated hearing before CI provision. ASR might be a useful tool for judging speech quality and can identify the need for speech or hearing rehabilitation in CI users.

\section{Acknowledgments}

Special thanks go to PD Dr. F. Schiel and V. Neumeyer of the Institute for Phonetics and Speech Processing of the University of Munich. Their support in the preparation and conduction of this investigation was valuable. Thanks also go to Naomi Zens for her help in writing the manuscript.

\section{Disclosure Statement}

The authors declare no conflict of interest in this work.

\section{References}

1 Leder SB, Spitzer JB: A perceptual evaluation of the speech of adventitiously deaf adult males. Ear Hear 1990; 11:169-175.

2 Svirsky MA, Sagi E, Meyer TA, Kaiser AR, Teoh WS: A mathematical model of medial consonant identification by cochlear implant users. J Audiol Soc Am 2011;129:2191-2200.

3 Müller J, Brill S, Hagen R, Moeltner A, Brockmeier SJ, Stark T, Helbig S, Maurer J, Zahnert T, Zierhofer C, Nopp $\mathrm{P}$, Anderson I: Clinical trial results with the MED-EL fine structure processing coding strategy in experienced cochlear implant users. ORL J Otorhinolaryngol Relat Spec 2012;74:185-198.

4 Kleine Punte A, De Bodt M, van de Heyning P: Long-term improvement of speech perception with the fine structure processing coding strategy in cochlear implants. ORL J Otorhinolaryngol Relat Spec 2014;76:36-43.

5 Stevens KN: Diverse acoustic cues at consonantal landmarks. Phonetica 2000;57:139-151.

6 Teoh SW, Neuburger HS, Svirsky MA: Acoustic and electrical pattern analysis of consonant perceptual cues used by cochlear implant users. Audiol Neurootol 2003;8:269-285.

7 Perkell J, Lane H, Svirsky M, Webster J: Speech of cochlear implant patients: a longitudinal study of vowel production. J Acoust Soc Am 1992;91:2961-2978.

8 Tye-Murray N, Kirk K: Vowel and diphthong production by young users of cochlear implants and the relationship between the phonetic level evaluation and spontaneous speech. J Speech Hear Res 1993;36:488-502. 
Ruff et al.: Speech Production Quality of Cochlear Implant Users with Respect to Duration and Onset of Hearing Loss

9 Matthies M, Svirsky M, Lane H, Perkell J: A preliminary study of the effects of cochlear implants on the production of sibilants. J Acoust Soc Am 1994;96:1367-1373.

10 Lane H, Wozniak J, Matthies M, Svirsky M, Perkell J: Phonemic resetting versus postural adjustments in the speech of cochlear implant users: an exploration of voice-onset time. J Acoust Soc Am 1995;98:3096-3106.

11 Lane H, Matthies M, Perkell J, Vick J, Zandipour M: The effects of changes in hearing status in cochlear implant users on the acoustic vowel space and CV coarticulation. J Speech Lang Hear Res 2001;44:552-563.

12 Perkell J, Numa W, Vick J, Lane H, Balkany T, Gould J: Language-specific, hearing-related changes in vowel spaces: a preliminary study of English- and Spanish-speaking cochlear implant users. Ear Hear 2001;22:461470.

13 Schenk B, Baumgartner W, Hamzavi J: Changes in vowel quality after cochlear implantation. ORL 2003;65: 184-188.

14 Lane H, Denny M, Guenther FH, Hanson HM, Marrone M, Matthies ML, Perkell JS, Stockmann E, Tiede M, Vick J, Zandipour M: On the structure of phoneme categories in listeners with cochlear implants. J Speech Lang Hear Res 2007;50:2-14.

15 Gould J, Lane H, Vick J, Perkell J, Matthies M, Zandipour M: Changes in speech intelligibility of postlingually deaf adults after cochlear implantation. Ear Hear 2001;22:453-460.

16 Langereis MC, Dejonckere PH, van Olphen AF, Smoorenburg GF: Effect of cochlear implantation on nasality in post-lingually deafened adults. Folia Phoniatr Logop 1997;49:308-314.

17 Neumeyer V, Harrington J, Draxler C: An acoustic analysis of the vowel space in young and old cochlearimplant speakers. Clin Linguist Phon 2010;24:734-741.

18 Horga D, Liker M: Voice and pronunciation of cochlear implant speakers. Clin Linguist Phon 2006;20:211-217.

19 Uchanski R, Geers A: Acoustic characteristics of the speech of young cochlear implant users: a comparison with normal-hearing age-mates. Ear Hear 2003;24(1 suppl):90S-105S.

20 Guenther FH, Ghosh SS, Tourville JA: Neural modeling and imaging of the cortical interactions underlying syllable production. Brain Lang 2006;96:280-301.

21 Menard L, Polak M, Denny M, Burton E, Lane H, Matthies ML, Marrone N, Perkell JS, Tiede M, Vick J: Interactions of speaking condition and auditory feedback on vowel production in postlingually deaf adults with cochlear implants. J Acoust Soc Am 2007;121:3790-3801.

22 Perkell JS, Zandipour M, Matthies ML, Lane H: Economy of effort in different speaking conditions. I. A preliminary study of intersubject differences and modeling issues. J Acoust Soc Am 2002;112:1627-1641.

23 Lazard DS, Vincent C, Venail F, van de Heyning P, Truy E, Sterkers O, Skarzynski PH, Skarzynski H, Schauwers K, O'Leary S, Mawman D, Maat B, Kleine-Punte A, Huber AM, Green K, Govaerts PJ, Fraysse B, Dowell R, Dillier N, Burke E, Beynon A, Bergeron F, Başkent D, Artières F, Blamey PJ: Pre-, per- and postoperative factors affecting performance of postlinguistically deaf adults using cochlear implants: a new conceptual model over time. PLoS One 2012;7:e48739.

24 Schiavetti N: Scaling procedures for the measurement of speech intelligibility; in: Intelligibility in Speech Disorders: Theory, Measurement and Management. Philadelphia, John Benjamins, 1992, pp 11-34.

25 Ziegler W, Hartmann E, Cramon DY: Word identification testing in the diagnostic evaluation of dysarthric speech. Clin Linguist Phon 1998;2:291-308.

26 Kitzing P, Maier A, Ahlander VL: Automatic speech recognition (ASR) and its use as a tool for assessment or therapy of voice, speech, and language disorders. Logoped Phoniatr Vocol 2009;34:91-96.

27 Windrich M, Maier A, Kohler R, Nöth E, Nkenke E, Eysholdt U, Schuster M: Automatic quantification of speech intelligibility of adults with oral squamous cell carcinoma. Folia Phoniatr Logop 2008;60:151-156.

28 Maier A, Haderlein T, Eysholdt U, Rosanowski F, Batliner A, Schuster M, Nöth E: PEAKS - a system for the automatic evaluation of voice and speech disorders. Speech Commun 2009;51:425-437.

29 Schukat-Talamazzini EG, Niemann H: Das ISADORA-System - ein akustisch-phonetisches Netzwerk zur automatischen Spracherkennung. Mustererkennung 1991;290:251-258.

30 Gallwitz F, Niemann H, Nöth E: Speech recognition - state of the art, application, and future prospects. Wirtschaftsinformatik 1999;41:538-547.

31 Schuster M, Haderlein T, Nöth E, Lohscheller J, Eysholdt U, Rosanowski F: Intelligibility of laryngectomees' substitute speech: automatic speech recognition and subjective rating. Eur Arch Otorhinolaryngol 2006;263: 188-193.

32 Schuster M, Maier A, Haderlein T, Nkenke E, Wohlleben U, Rosanowski F, Eysholdt U, Nöth E: Evaluation of speech intelligibility for children with cleft lip and palate by means of automatic speech recognition. Int J Pediatr Otorhinolaryngol 2006;70:1741-1747.

33 Haderlein T, Eysholdt U, Riedhammer K, Nöth E, Rosanowski F: Automatisierung des PostlaryngektomieTelefontests (PLTT); in Gross M, Kruse E (eds): Aktuelle phoniatrisch-pädaudiologische Aspekte 2007. Norderstedt, Books on Demand GmbH, 2007, pp 66-69.

34 Cosetti M, Roland JT Jr: Cochlear implantation in the very young child: issues unique to the under-1 population. Trends Amplif 2010;14:46-57.

35 Mayr S, Burkhardt K, Schuster M, Maier A, Rogler K, Iro H: The use of automatic speech recognition showing the influence of nasality on speech intelligibility. Eur Arch Otorhinolaryngol 2010;267:1719-1725.

36 Scherf F, van Deun L, van Wieringen A, Wouters J, Desloovere C, Dhooge I, Offeciers E, Deggouj N, De Raeve L, Wuyts FL, van de Heyning P: Subjective benefits of sequential bilateral cochlear implantation in young children after 18 months of implant use. ORL J Otorhinolaryngol Relat Spec 2009;71:112-121. 
Ruff et al.: Speech Production Quality of Cochlear Implant Users with Respect to Duration and Onset of Hearing Loss

37 Paal S, Reulbach U, Strobel-Schwarthoff K, Nkenke E, Schuster M: Evaluation of speech disorders in children with cleft lip and palate. J Orofac Orthop 2005;66:270-278.

38 Maier A, Höning F, Bocklet T, Nöth E, Stelzle F, Nkenke E, Schuster M: Automatic detection of articulation disorders in children with cleft lip and palate. J Acoust Soc Am 2009;126:2589-2602.

39 Brown KD, Balkany TJ: Benefits of bilateral cochlear implantation: a review. Curr Opin Otolaryngol Head Neck Surg 2007;15:315-318.

40 Culling JF, Jelfs S, Talbert A, Grange JA, Backhouse SS: The benefit of bilateral versus unilateral cochlear implantation to speech intelligibility in noise. Ear Hear 2012;33:673-682.

41 Finley C, Holden T, Holden L, Whiting B, Chole R, Neely G, Hullar T, Skinner M: Role of electrode placement as a contributor to variability in cochlear implant outcomes. Otol Neurotol 2008;29:920-928.

42 Berrettini S, Forli F, Passetti S: Preservation of residual hearing following cochlear implantation: comparison between three surgical techniques. J Laryngol Otol 2008;122:246-252.

43 Miranda P, Sampaio A, Lopes R, Ramos Venosa A, de Oliveira C: Hearing preservation in cochlear implant surgery. Int J Otolaryngol 2014;2014:468515.

44 An YS, Kim ST, Chung JW: Preoperative voice parameters affect the postoperative speech intelligibility in patients with cochlear implantation. Clin Exp Otorhinolaryngol 2012;5:69-72.

45 Gordon KA, Wong DD, Valero J, Jewell SF, Yoo P, Papsin BC: Use it or lose it? Lessons learned from the developing brains of children who are deaf and use cochlear implants to hear. Brain Topogr 2011;24:204-219.

46 Petersen B, Gjedde A, Wallentin M, Vuust P: Cortical plasticity after cochlear implantation. Neural Plast 2013; 2013:318521.

47 Wilpon JG, Jacobsen CN: A study of speech recognition for children and the elderly. ICASSP '96 Proceedings of Acoustics, Speech, and Signal Processing 1996;1:349-352.

48 Peng SC, Tomblin JB, Spencer LJ, Hurtig RR: Imitative production of rising speech intonation in pediatric cochlear implant recipients. J Speech Lang Hear Res 2007;50:1210-1227.

49 Tanamati LF, Bevilacqua MC, Costa OA: Cochlear implant in postlingual children: functional results 10 years after the surgery. Braz J Otorhinolaryngol 2012;78:103-110. 